\title{
A new concept in distal arch aneurysm repair with a stent graft
}

Yoshifumi Iguro, MD, Kenichi Arata, MD, Hirouki Yamamoto, MD, Hiroshi Masuda, MD, and Ryuzo Sakata, MD, Kagoshima, Japan

$\mathrm{T}$

ranscatheter replacement by a stent graft to the angulated aortic segments with critical side branches carries risk of serious complications. In an attempt to overcome such problems, we have developed a novel branched stent

From the Second Department of Surgery, Kagoshima University, Faculty of Medicine, Kagoshima, Japan.

Received for publication June 12, 2001; accepted for publication Aug 1, 2001.

Address for reprints: The Second Department of Surgery, Kagoshima University, Faculty of Medicine, 8-35-1, Sakuragaoka, Kagoshima City, Kagoshima Prefecture, 890-8520 Japan (E-mail: iguro@med6.kufm.kagoshima-u.ac.jp).

J Thorac Cardiovasc Surg 2002;123:378-80

Copyright (C) 2002 by The American Association for Thoracic Surgery

$0022-5223 / 2002 \$ 35.00+0 \quad \mathbf{1 2 / 5 4 / 1 1 9 3 3 0}$

doi: $10.1067 / \mathrm{mtc} .2002 .119330$ graft that can be used to simultaneously reconstruct the left subclavian artery and the distal aortic arch. This article details the first use of such an implant.

\section{Clinical Summary}

A 64-year-old man was admitted to our institute with hoarseness due to a thoracic aortic aneurysm. The aneurysm's maximum diameter was $70 \mathrm{~mm}$, which represented an increase of $25 \mathrm{~mm}$ from 18 months earlier. A computed tomogram and an aortogram revealed a large saccular aneurysm with mural thrombus in the distal aortic arch and left subclavian artery (Figure 1, $A$ and $B$ ). The patient had previously received an axillo-bifemoral bypass, aortobifemoral bypass, and left femoro-popliteal bypass for other atherosclerotic lesions. Previous surgical interventions included treatment for dilated cardiomyopathy. Additional comorbidity factors included pronounced dilation and poor contraction of the left ventricle, both of which were diagnosed by echocardiography (end- 

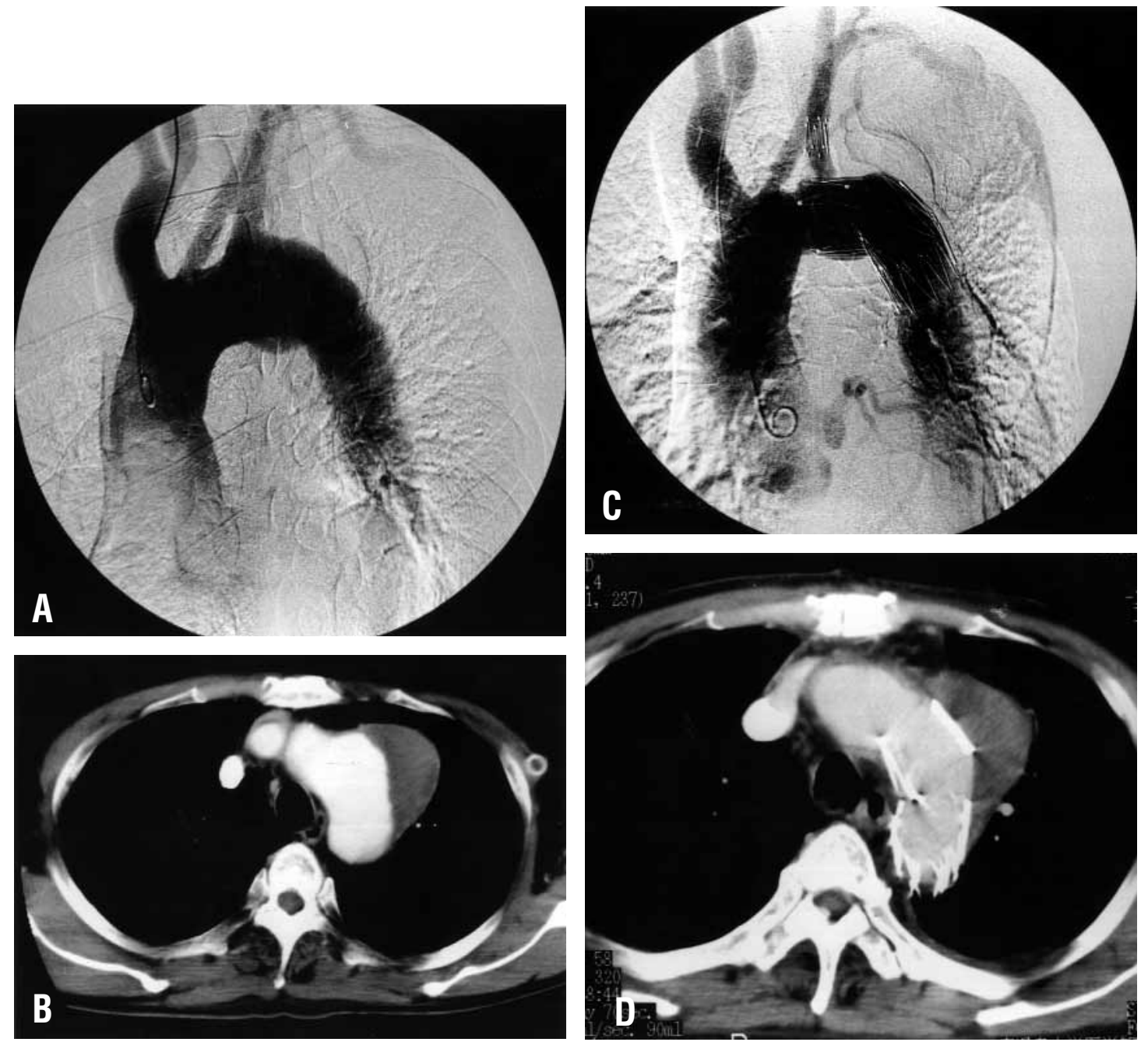

Figure 1. Preoperative aortogram and computed tomogram. A, Preoperative aortogram shows the saccular projection in the distal aortic arch involving the left subclavian artery. B, Enhanced computed tomogram shows the large saccular aneurysm with thrombus in the distal aortic arch. C, Postoperative aortogram (2 weeks after the operation) shows the disappearance of the sacral aneurysm and the normal flow through the aortic arch and the left subclavian artery. D, Computed tomogram (10 days after the operation) shows complete clot formation of the aneurysm and no leakage.

diastolic/end-systolic left ventricular dimension $=69 / 60$, percent fractional shortening $=13 \%$, ejection fraction $=27 \%$ ).

Cardiopulmonary bypass was initiated through venous drainage of the right atrium and arterial delivery to the ascending aorta. The left ventricle was then vented through the right superior pulmonary vein. The patient was cooled until the rectal temperature reached $25^{\circ} \mathrm{C}$. Cardiac arrest was induced through antegrade blood cardioplegia. After circulatory arrest was established, the aortic arch was opened transversely between the left carotid artery and the left subclavian artery. Selective antegrade cerebral perfusion was performed at a rate of $600 \mathrm{~mL} / \mathrm{min}$. Our stent graft consisted of a stem and one branch graft. The stem portion was constructed from a selfexpanding 40-mm Gianturco stainless-steel Z stent (Cook, Inc, Bloomington, Ind) and a thin-walled 36-mm woven Dacron vascu- lar graft (Ubekosan, Inc, Ube, Japan). The length of the stem intended for the aortic arch was $100 \mathrm{~mm}$, with the middle segment of the prosthesis free of the stent to facilitate flexibility, allowing it to be tailored to the curvature of the distal aortic arch. The branch graft for the left subclavian artery was constructed from a thinwalled 12-mm woven Dacron vascular graft and a 12-mm stainlesssteel spiral stent (Figure 2). The branch graft was approximately 27 $\mathrm{mm}$ long, and the spiral stent was fixed to the free end of the graft. A forceps shaped to match the curve of the aortic arch was used to maneuver the stem graft to the intended distal anastomotic portion via the proximal incision site. Endoscopy and transesophageal echocardiography were used to deploy the prosthesis at a site that excluded the aneurysmal lesion. After stem graft placement, the branch graft was inserted into the left subclavian artery with right- 


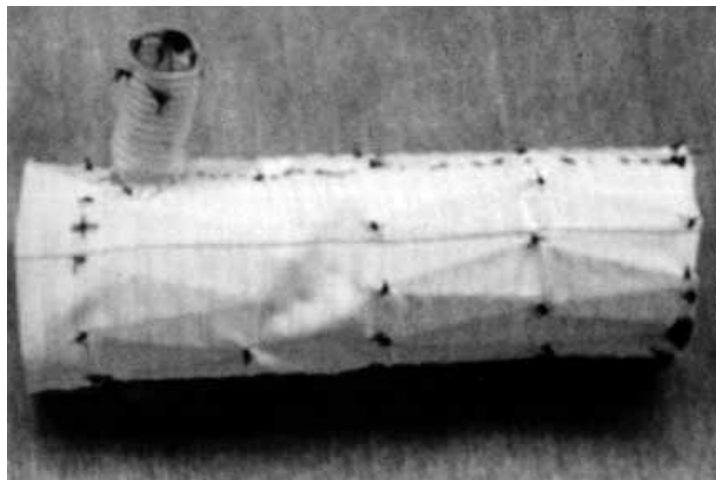

Figure 2. Flexible stent graft with one branch. The middle segment of the stent graft is free of the stent, increasing its flexibility. One branch consists of a 12-mm spiral stent and a thin-walled graft.

angled forceps and was then fixed to the base of the subclavian artery at the branch point with a 4-0 Prolene suture (Ethicon, Inc, Somerville, NJ). The proximal end of the stent graft was fixed to the aorta circumferentially using a 4-0 Prolene suture running just proximal to the entrance of the left subclavian artery. The aortotomy was then closed. Operation time, selective cerebral perfusion time, and cardiopulmonary bypass time were 295 minutes, 81 minutes, and 191 minutes, respectively. The patient had an uneventful recovery and was discharged from the intensive care unit within 2 days. An aortogram performed 2 weeks after the operation revealed the complete obliteration of the aneurysm and undisturbed flow through the aortic arch, the left subclavian artery, and the graft site. A computed tomogram performed 10 days after the operation showed complete clot formation in the excluded aneurysm (Figure
1, $C$ and $D$ ). During the 15-month follow-up period, the patient was free of symptoms, and he remains in good health.

\section{Comment}

Transcatheter stent graft repair of distal arch aneurysm involving the left subclavian artery is difficult because of the presence of curved and branch vessels. ${ }^{1}$ Kato and colleagues ${ }^{2}$ have reported a new implantation technique that uses a stent graft for thoracic aortic aneurysm. They repaired a distal arch aneurysm using a stent graft inserted through the aortic arch during an ascending aortoaxillary bypass. The use of this bypass technique in the reconstruction of the left subclavian artery requires the use of a side clamp during anastomosis of the ascending aorta. This, in turn, carries the risk of atheromatous plaques. The fact that our stent graft includes a branch for the left subclavian artery obviated the need for an extra-anatomic bypass and thus reduced the risk of mural thrombus.

To date we have used this technique in 4 patients exhibiting distal arch aneurysm, all with favorable outcomes. We therefore recommend that this technique be considered an option for surgical intervention when dealing with a distal arch aneurysm that includes subclavian branch repair. We believe that this technique will become increasingly feasible and that a broad range of applications will be developed for total arch repair.

\section{References}

1. Dake MD, Miller DC, Mitchell RS, Semba CP, Moore KA, Sakai T. The "first generation" of endovascular stent-grafts for patients with aneurysms of the descending thoracic aorta. $J$ Thorac Cardiovasc Surg. 1998;116:689-704.

2. Kato M, Kaneko M, Kuratani T, Horiguchi K, Ikushima H, Ohnishi K. New operative method for distal aortic arch aneurysm: combined cervical branch bypass and endovascular stent-graft implantation. $J$ Thorac Cardiovasc Surg. 1999;117:832-4. 